\title{
DESARROLLO Y ACTUALIDAD DE LA PROTECCIÓN SOCIAL EN AMÉRICA LATINA
}

\author{
Silvia Gabriela Fernández Soto \\ Universidad Nacional del Centro de la Provincia de Buenos Aires (UNCPBA)
}

\section{DESARROLLO Y ACTUALIDAD DE LA PROTECCIÓN SOCIAL EN AMÉRICA LATINA}

Resumen: El presente trabajo tiene como objetivo problematizar las recientes estrategias de protección social en América Latina, observando la expansión e institucionalización de los programas de transferencia de renta condicionada. Se basa en un estudio exploratorio, bibliográfico y documental. Es en el contexto de crisis y conflictividad social de fines de siglo $\mathrm{XX}$ y principios de siglo XXI, en un contexto de desocupación y extensión de las condiciones precarias de trabajo; que cobran relevancia los Programas de Transferencias de Renta Condicionadas (PTRC). Se han instalado en el repertorio de políticas sociales desarrollados por la mayoría de los países en América Latina para el contingente creciente de desocupados y pobres, configurando estrategias político- institucionales que se hacen masivas a inicios del Siglo XXI, institucionalizándose en los formatos de protección social emergentes. Estos programas se identifican por estar focalizados hacia los hogares pobres, con el objetivo de mejorar las condiciones de vida de las familias con niños y niñas en "situación de vulnerabilidad", con una perspectiva de "capital humano", incluyendo condicionalidades en educación y salud. Se enmarcan en el debate de la protección social centrada en un tipo de "universalismo" adjetivado como "mínimo/básico", que pretende romper el "círculo intergeneracional de la pobreza". Analizamos el sentido socio-histórico que adquieren estos programas en relación a transformaciones sociales más generales.

Palabras clave: Protección social, crisis, Transferencia de Renta Condicionada.

\section{DEVELOPMENT AND SOCIAL PROTECTION NEWS IN LATIN AMERICA}

Abstract: The objective of this work is to problematize the recent strategies of social protection in Latin America, noting the expansion and institutionalization of the income transfer conditional programs. It is based on an exploratory study, bibliographic and documentary. It is in the context of crisis and social unrest of the late twentieth century and early twentyfirst century, in a context of unemployment and extension of the precarious working conditions; that prominence programs of income transfers conditioned (PTRC). Have been installed in the repertoire of social policies developed by the majority of the countries in Latin America to the growing contingent of unemployed and poor, setting up political-institutional strategies that are massive at the beginning of the twenty-first century, institutionalized in the formats of emerging social protection. These programs are identified as being targeted toward poor households, with the aim of improving the living conditions of families with children in "situation of vulnerability", with a perspective of "human capital", including conditionalities in education and health.and that they fit into the debate of social protection focused on a kind of "universalism" adjective as "minimum / basic", which aims to break the "intergenerational cycle of poverty." It also analyzes the socio-historical sense that purchase these programs in relation to more general social transformations

Key words: Social protection, crisis, Income Transfer Programs. 
1 LA PROTECCIÓN SOCIAL COMO PROCESOS HISTÓRICOS CONTRADICTORIOS: la(s) experiencia(s) en América Latina

La construcción histórica de las formas de protección social remite a complejos procesos relacionales en los cuales participan múltiples elementos. Por lo tanto no se puede restringir su comprensión a identificar un único proceso evolutivo lineal, incremental, simplificado y abstracto. Existen en América Latina diferentes experiencias en la construcción de los sistemas de protección social, que expresan movimientos progresivos y regresivos en función de las relaciones de poder en las que se inscriben en cada formación histórico-concreta.

Desde el punto de vista de la economía política de la configuración del Estado, la extensión de los sistemas de protección social, y la mediación de las políticas sociales que suponen; participan al mismo tiempo de las necesidades de acumulación capitalista, y de las necesidades de protección y reproducción de las condiciones materiales de existencia de los trabajadores ${ }^{2}$. En este sentido, los formatos de protección social adquieren concreción en contextos particulares de acumulación y de procesamiento de intereses antagónicos entre las clases sociales fundamentales. En tal sentido expresan en cada momento histórico correlaciones de fuerza, y por ende la organización y capacidad de presión de los trabajadores.

En términos histórico-concretos la construcción de los sistemas de protección social estatal se vincula a garantizar los procesos de acumulación y legitimación e indica la relación histórica que se establece con los procesos de organización del trabajo. Participa en la reproducción y manutención de la clase trabajadora (activa y parada), a través de mediaciones políticoinstitucionales específicas, que son resultado de la correlación de fuerzas establecida por la lucha de clases. Desde esta perspectiva, la tesis que sostenemos es que en el contexto de crisis que se expresa desde la década del setenta y el despliegue de la acumulación flexible, en un contexto general regresivo para los intereses de las clases trabajadoras, se alteran los formatos y sentidos de los procesos de protección social históricamente construidos en la segunda posguerra del siglo $\mathrm{XX}$, edificándose nuevas arquitecturas de protección social, resultantes de las nuevas relaciones entre acumulación y organización del trabajo.

En las diversas naciones latinoamericanas se registran variaciones en los sistemas de protección social; los cuales están en relación a las características de los procesos de acumulación/ legitimación y de las heterogeneidades nacionales concretas $^{3}$. La concreción particular de un conjunto de elementos históricos da como resultado la disposición de diferentes matrices de protección social, observable en el gasto social y su distribución sectorial, en la cobertura poblacional, en la forma y contenido de los servicios prestados, en la calidad de los mismos, en los argumentos de legitimación que se construyen para sostenerlos, en los sentidos que persiguen, en las pautas de selectividad que sostienen, en los intereses y sujetos a los cuales responden.

En América Latina en la década de $1930^{4}$ gana visibilidad formas de protección social destinada centralmente a los trabajadores urbanos y asalariados insertos en relaciones formales de trabajo. Bajo esta perspectiva la estructuración del mercado de trabajo influyó los sistemas de protección social, marcando las lógicas de inclusión/exclusión de los mismos, así como las diferenciaciones y fragmentaciones que se establecen. Es en la segunda posguerra que se consolidan estas experiencias particulares ${ }^{5}$, guiados por los principios de los seguros a los riesgos colectivos, bajo el esquema contributivo y con la fuerte participación del Estado como garante de la protección en su gestión y financiamiento. Ahora bien, como indica Filgueira (2007) América Latina no se configuró como las experiencias europeas en relación a sus procesos de urbanización, industrialización, con modelos familiares nucleares tradicionales y con mercados laborales formales, presupuestos bajo los cuales se definieron las políticas sociales latinoamericanas en el pasado, aspirando a que los grandes centros urbanos se acerquen a dichas experiencias. No sólo esto no sucedió, sino que se despliegan un conjunto de fenómenos que distan de esta realidad.

\begin{abstract}
Así,elcrecimientodelamonoparentalidad y las uniones libres, la incorporación masiva de la mujer al mercado laboral, el incremento de la divorcialidad, son realidades innegables en la mayor parte de la región. Asimismo, la creciente precariedad en el empleo, el crecimiento de la informalidad y de la flexibilidad contractual en los viejos formatos de trabajo estable, la desindustrialización y el crecimiento de las economías de servicios, acompañados de una transformación tecnológica que torna obsoletos saberes a una velocidad desconocida en la región, son también procesos de clara implantación en las últimas dos décadas. (FILGUEIRA, 2007, p. 15).
\end{abstract}

Que sin duda interpelan y complejizan los sistemas de protección social. Este esquema implicó que no se desarrolle una cobertura total de la población, que se constituyan altamente fragmentado y estratificado de acuerdo a la inserción ocupacional de la población; segmentado en función de las disparidades de cobertura a nivel regional, produciéndose al interior del sistema fuertes disparidades de acceso a los bienes y servicios públicos tanto en calidad como en cantidad. 
Otros estudios, además de considerar cuándo surgen los sistemas de protección y los alcances que tienen en materia de cobertura y el nivel de gasto que realizan; consideran central observar no sólo cuánto se gasta sino cómo se gasta en materia social $^{6}$. En relación al primer grupo, universalismo estratificado, cabe destacar que hacia 1970 todos protegían de alguna u otra forma a la mayor parte de la población mediante sistemas de seguro social, de servicios de salud al mismo tiempo habían extendido la educación primaria e inicial secundaria a toda la población. Estos países desarrollan importantes procesos de desmercantilización y universalización, aunque de manera estratificada y diferencial. Logran importantes impactos sobre variables relativas al bienestar social. En relación a los regímenes duales presentaban hasta la década de los '70 universalización de la educación primaria, una importante cobertura en salud aunque estratificada. En materia de seguridad social alcanza coberturas medias, manteniendo también significativos niveles de estratificación. Un rasgo central refiere a la heterogeneidad territorial, que se presenta con mayor nitidez que el primer grupo. En ciertos estados y regiones se observa la maduración de instituciones de protección social y en cambio en otros estados desprotección y baja incorporación de la mayor parte de la población.

En el contexto de la crisis capitalista desplegada desde la década del setenta los formatos concretos de protección social construidos en los diferentes países sufren transformaciones radicales en relación a sus sentidos, principios organizadores, diseños de implementación y alcances. Estas transformaciones están en relación a las exigencias de clase colocadas al Estado en la fase actual de acumulación capitalista bajo el predominio del capital financiero. Acumulación y legitimación se recrean en complejos procesos en relación a la correlación de fuerzas resultantes del desarrollo de la sociedad. Observar los cambios generales nos permite comprender los sentidos de las reformas de la protección social en este contexto de crisis capitalista.

\section{CRISIS CAPITALISTA Y TRANSFORMACIONES DE LOS SISTEMAS DE PROTECCIÓN SOCIAL EN EL CONTEXTO LATINOAMERICANO}

La crisis capitalista que se inicia hacia fines de la década del sesenta e inicios de la década del 70 del siglo pasado, expresa el colapso del padrón de acumulación fordista/keynesiano; dando origen a un período en la historia del capital en donde se da en forma exacerbada la destrucción de las fuerzas productivas, de la naturaleza y del medio ambiente y también de la fuerza humana del trabajo ${ }^{7}$. Es en el contexto de acumulación fordista que se desplegaron los modelos de desarrollo ${ }^{8}$ de inspiración keynesiana ${ }^{9}$ en gran parte de la región latinoamericana entre 1930 y 1970-80, caracterizados por el modelo sustitutivo de importaciones, donde el Estado asumió un rol central en el desarrollo económico y en la construcción de los sistemas de protección social. La respuesta capitalista a la crisis de mediados de la década del setenta implica la alteración de estos modelos de desarrollo y las correlaciones de fuerza que los sustentan.

Desde 1970 se expresan un conjunto de cambios radicales en la organización del capitalismo: Se produce un pasaje del modelo de acumulación de capital fordista (cadena de montaje de producción en masa, organización política de masas, intervenciones del Estado de Bienestar), a la acumulación flexible (búsqueda y configuración de mercados especializados, descentralización y dispersión espacial de la producción, retracción del Estado Nación de las políticas intervencionistas unida a la liberalización y la privatización) (HARVEY, 2007). La crisis y reestructuración capitalista a escala global, genera una re-configuración territorial, socio- económica y política a escala global. Estas transformaciones expresan claramente la contestación rotunda del capital a la caída de la tasa de ganancia en las décadas del sesenta y setenta del siglo XX. Es así que los años siguientes se caracterizaron por transformaciones radicales en el plano tecnológico y organizacional del proceso productivo $^{10}$.

Esta fase de acumulación flexible, se caracteriza por los elevados niveles de transnacionalización de las empresas capitalistas, la mundialización de las relaciones capitalistas de producción, el desarrollo y tecnificación del capital financiero (HARVEY, 2004; 2007) $)^{11}$.

En el marco de los denominados ajustes estructurales, bajo la influencia de los organismos internacionales, se le otorga un nuevo perfil a los sistemas de protección social y a las políticas diseñadas por los Estado nacionales, que termina conformando una nueva matriz de relaciones entre el Estado y la sociedad civil.

Dentro de estas transformaciones se destaca la relevancia que las redes mínimas de asistencia centradas en la pobreza pretenden asumir en las últimas décadas, en contraposición a la desestructuración de la red de protección social tendida en relación al asalariado formal desde la perspectiva del seguro social.

Las propuestas y tendencias centrales que se impone se orientaron en la modificación de las políticas de protección social en la región, hacia una perspectiva residual-liberal; tanto en los países que lograron desarrollos significativos bajo esquemas centralistas con aspiraciones de universalidad, como en aquellos países que tuvieron bajo o nulo desarrollo de los sistemas de protección social. La focalización del gasto, la descentralización/desconcentración 
en la gestión y administración de los programas sociales, el desdibujamiento de la perspectiva sectorial de las políticas sociales y el avance de la integralidad mínima como alternativa, y la expansión de la mercantilización de ciertas funciones sociales, constituyen los pilares de las reformas y nuevos modelos propuestos en las últimas décadas del siglo $\mathrm{XX}$.

\section{EXPANSIÓNDELAPOBREZAY DESIGUALDAD} A FINES DE SIGLO XX: inflexiones y ejes estructurales de desigualdad social a inicios del Siglo XXI

En torno a 1999 , el $43.8 \%$ de la población de la región se encontraba en situación de pobreza, tres décimas de punto más que en 1997. Por su parte, la indigencia pasó del $19 \%$ al $18.5 \%$ en ese período. En términos del volumen de población en situación de pobreza, éste alcanzaba en 1999 a poco más de 211 millones de personas, de las cuales algo más de 89 millones se encontraban bajo la línea de indigencia. Con respecto a 1997 esta cifra representa un aumento de 7.6 millones de personas pobres, en tanto que el número de indigentes tuvo un incremento en el trienio, que abarcó a 0.6 millones de personas.

Para 1999, la desigual distribución de los ingresos continuaba siendo un rasgo sobresaliente de la estructura económica y social de América Latina, lo que le ha valido ser considerada la región menos equitativa del mundo, empeorando esta situación en la década del noventa. Para fines del siglo XX, la distribución del ingreso en América Latina exhibe importantes niveles de desigualdad; la fracción de los ingresos totales que reúne el $10 \%$ de los hogares de mayores recursos (salvo en Costa Rica y Uruguay) asciende en todos los países de la región a más del $30 \%$ de los ingresos, y en la mayoría de ellos ese porcentaje supera el $35 \%$. En contraposición, la fracción del ingreso recibida por el $40 \%$ de los hogares más pobres es muy reducida, y se ubican en casi todos los países entre el $9 \%$ y el $15 \%$ de los ingresos totales, con excepción de Uruguay donde el grupo mencionado recibe cerca del $22 \%$.

En este marco de empobrecimiento e incremento de la desigualdad, América Latina se coloca como uno de los territorios de resistencias y búsquedas de alternativas al capitalismo neoliberal a nivel global. La lucha de clases expresaba la oposición al ajuste estructural impuesto por los organismos internacionales, las actividades depredadoras del capital financiero y la pérdida de derechos ocasionada por la privatización y mercantilización de los servicios sociales. "El antimperialismo comenzó a convertirse en antagonismo contra los principales agentes de la financierización, el FMI, y el Banco Mundial." (HARVEY, 2007, p. 65). Este movimiento ascendente de las luchas sociales provoca inflexiones y exige la recomposición del consenso.

América Latina en la primera década del siglo XXI exhibe inflexiones en relación a las situaciones socioeconómicas registradas en las décadas anteriores del 80 y 90 . Pese a estas mejoras en los indicadores permanecen enormes deudas sociales.

Se registra una disminución del porcentaje de las personas que viven en condiciones de pobreza e indigencia. La reducción de la pobreza acumulada desde 1999 alcanzó 12.4\%, a la vez que la indigencia se ha reducido un $6.3 \%$. Asimismo, la reducción de ambos indicadores con respecto a 1990 totalizaba 17.0 y 10.3\% (COMISIÓN ECONÓMICA PARA AMÉRICA LATINA Y EL CARIBE, 2011). En 2010, el índice de pobreza de la región se situó en un $31,4 \%$, lo que incluye a un $12,3 \%$ de personas en condiciones de pobreza extrema o indigencia. En términos absolutos, estas cifras equivalen a 177 millones de personas pobres, de las cuales 70 millones eran indigentes (COMISIÓN ECONÓMICA PARA AMÉRICA LATINA Y EL CARIBE, 2011). Si bien en términos relativos se registra una disminución, en términos absolutos significa un incremento de 41 millones de pobres entre 1980 y 2010; y un incremento de 8 millones de indigentes para el mismo período (Gráfico 1). Esto expresa que un tercio de los habitantes de la región, no reciben ingresos suficientes para cubrir las necesidades consideradas básicas ${ }^{12}$. Pese a las mínimas variaciones observadas en términos de tasas, las nuevas estimaciones permiten establecer que la pobreza extrema ha alcanzado valores similares a los de 2011, lo que representa un retroceso respecto de los logros alcanzados en años anteriores. La tendencia a la baja de las tasas de pobreza y pobreza extrema se ha desacelerado e incluso revertido en los primeros años de la segunda década, situación que, asociada al crecimiento demográfico, deja como saldo un mayor número depersonas en situación de pobreza extrema en 2013 (COMISIÓN ECONÓMICA PARA AMÉRICA LATINA Y EL CARIBE, 2014).

El año 2010 se caracterizó por un aumento de las tasas de inflación en todos los países de la región. El promedio simple de las variaciones se situó en un 6,5\%, 2,8 puntos porcentuales más que en 2009 (COMISIÓN ECONÓMICA PARA AMÉRICA LATINA Y EL CARIBE, 2011). El aumento de la inflación estuvo impulsado centralmente por alzas de los precios de alimentos y bebidas, que en promedio fue 1,8 veces mayor que las del resto de productos, aspecto gravitante para la medición de la indigencia. Las proyecciones realizadas indican que se mantenga la tendencia al alza de la inflación, con lo cual la indigencia no sólo se mantendrá en los niveles registrados sino que puede aumentar ${ }^{13 .}$

Tanto en el período 2002-2008, previo a la crisis, como en el período 2008-2010, la reducción 
Gráfico 1 - América Latina: evolución de la pobreza y de la indigencia, 1980-2014
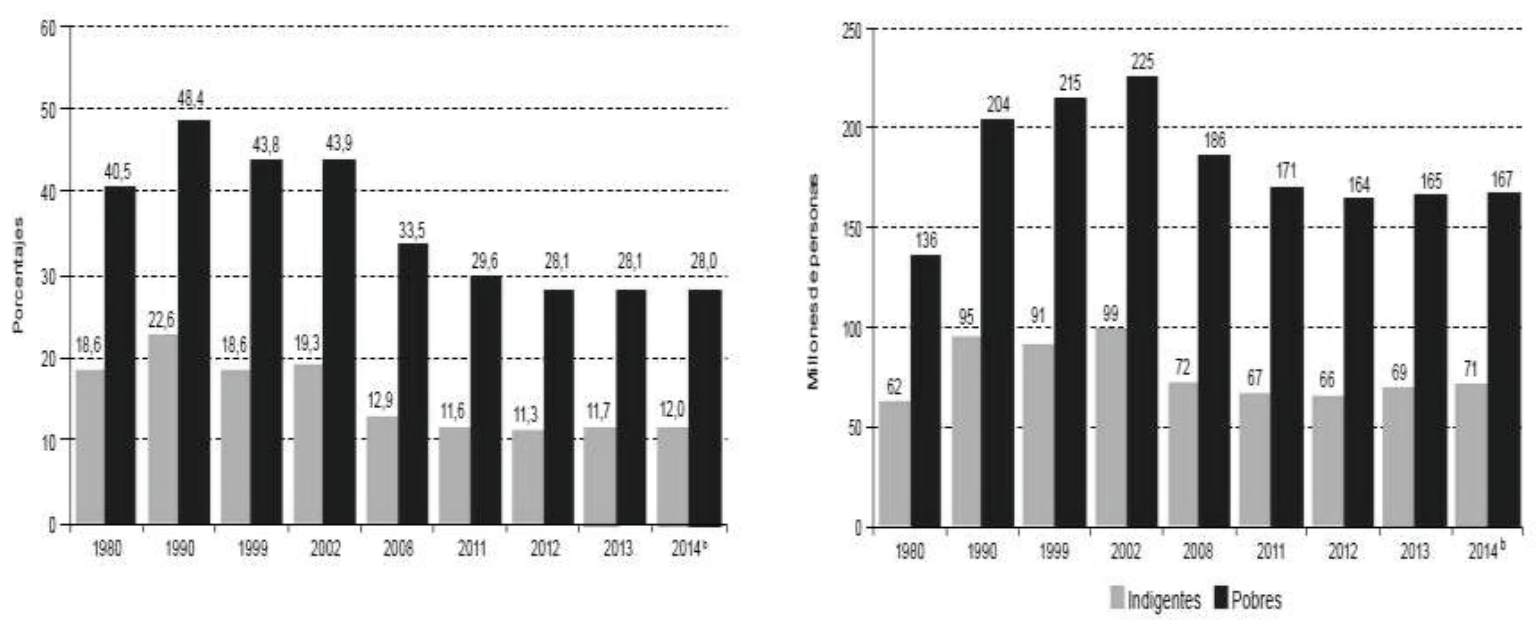

Fuente: Comision Eonnomica para America Latinay y Carbe ICEPAL, score la base de tabulaciones escecides de las encuestas de hogares de los respectios palises. - Estimacion correspondiente a 19 pases de America Latina, incuido Hati. No se inclyye a Cuba.

b Proyecoion.

de la pobreza ha provenido en su mayor parte de un incremento de los ingresos laborales; aunque también han contribuido otras fuentes de ingreso, particularmente las transferencias monetarias.

Si bien la reducción de la desigualdad es de una magnitud leve, insuficiente para cambiar la base estructural de desigualdad de la región, resulta significativa, en un contexto de ausencia prolongada de mejoras distributivas generalizadas ${ }^{14}$. Los datos más recientes arrojan algunos indicios que apuntan a que la reducción de la desigualdad podría estarse desacelerando (COMISIÓN ECONÓMICA PARA AMÉRICA LATINA Y EL CARIBE, 2014). Para el 20111 , el $40 \%$ de la población con los ingresos más bajos capta, en promedio, el 15\% del total del ingreso, mientras que el $10 \%$ de la población situado en el extremo superior de la distribución posee un tercio del ingreso total. Asimismo, el ingreso medio del quintil más rico supera en 18,3 veces al del quintil más pobre. América Latina continúa siendo una del las regiones del mundo más desigual ${ }^{15}$.

América latina y el Caribe cierran el 2011 con crecimiento económico ${ }^{16}$, crecimiento del empleo y disminución del desempleo (ORGANIZAÇÃO INTERNACIONAL DO TRABALHO, 2011).

La tasa de desempleo urbano continuó bajando en 2011 y alcanzó a fines de 2011 un nivel de $6,8 \%$, valores similares a los registrados en la década del 90. La región entró al Siglo XXI con tasas superiores al $10 \%$ que llegaron incluso por encima del $13 \%$. Lo que vemos ahora es un reflejo de un ciclo positivo de crecimiento económico que ha durado más de cinco años y no se vio interrumpido por la crisis.

Si bien el desempleo ha bajado, la proporción de trabajadores por cuenta propia y auxiliares en actividades de baja productividad sigue alta, cerca de un tercio del total del empleo en la región. Al mismo tiempo $44 \%$ de los trabajadores y trabajadoras aún no tienen ningún tipo de cobertura de protección social. En 16 países con información disponible hacia fines de la década del 2000, 93 millones de personas ( $50 \%$ de la población ocupada) tenían un empleo informal. De ese total, 60 millones estaban en la economía informal propiamente dicha, 23 millones tenían un empleo informal sin protección social trabajando en el sector formal, y 10 millones un empleo informal en el servicio doméstico. En el caso de los jóvenes, 6 de cada 10 que consiguen trabajo sólo tienen acceso a empleos informales (ORGANIZAÇÃO INTERNACIONAL DO TRABALHO, 2011).

La dinámica de la oferta y demanda de fuerza de trabajo, se pone en relación con la dinámica del ciclo económico de los países, explica la tendencia a la disminución de la tasa de desempleo urbano, que cae desde dos dígitos a inicios de la década a $7.3 \%$ en 2008 , sube a $8.1 \%$ en 2009 y baja a $7.3 \%$ en 2010 . El crecimiento económico permitió una expansión del empleo asalariado, que en el promedio regional (y con datos de cobertura nacional) aumentó su peso relativo en tres puntos porcentuales (de 65\% a $68 \%$ ). Pese a este crecimiento y a esta expansión registrada de trabajo asalariado, al finalizar la década cerca de uno de cada tres ocupados en la región son trabajadores por cuenta propia y auxiliares. La mayoría de estos se desempeña en actividades del sector informal, en condiciones de precariedad laboral, desprotección social y con baja productividad e ingresos.

La incorporación precaria de contingentes de trabajadores, es un factor determinante de los procesos de pobreza y empobrecimiento de la clase trabajadora y de perpetuación de las desigualdades sociales. Los datos indican la permanencia estructural de situaciones de precariedad laboral 
extendidas: puestos de trabajo de baja productividad, bajas remuneraciones y signados por la inestabilidad laboral, la desprotección social y la falta de acceso a los sistemas de seguridad social.

Esto nos indica que el crecimiento sostenido del Producto Interno Bruto (PIB) no significó la creación de suficientes empleos formales para reducir significativamente el empleo precario. La reducción no ha acompañado el crecimiento sostenido del PBI. Es decir, el alto crecimiento económico sostenido en estos años (principalmente entre 2003-2008) no se ha traducido en la superación de la alta informalidad de su mercado laboral. A pesar de que en 2010 la recuperación económica influyó positivamente en el funcionamiento del mercado de trabajo, con un incremento del índice de ocupación y una caída del desempleo, el mundo laboral continúa siendo en América Latina uno de los principales eslabones en la reproducción de la desigualdad. La heterogeneidad de la estructura productiva se expresa en una dispar polarización, por una parte, un sector minoritario, con empleos de alta productividad, salarios y protección social, y por otra, un sector donde predominan las condiciones laborales precarias, las remuneraciones más bajas y un limitado acceso a la protección social. Además, tanto el desempleo como la ocupación en el sector de baja productividad siguen afectando sobre todo a los jóvenes y a las mujeres más pobres.

\section{EMERGENCIA, INSTITUCIONALIZACIÓN Y CONSOLIDACIÓN DE LOS PROGRAMAS DE TRANSFERENCIAS DE RENTA CONDICIONADAS (PTRC)}

Es en el proceso global de redimensionamiento de los Sistemas de Protección Social por los cambios sociales generales y las nuevas exigencias colocadas al Estado, que se inscribe la emergencia y ampliación de los Programas de Transferencias de Renta Condicionadas (PTRC) alcanzando a los contingentes de trabajadores desocupados, empobrecidos, precarios e informales que no alcanzan a reproducir sus condiciones materiales de existencia.

Su emergencia, expansión y consolidación se relacionan en términos amplios con: 1 - el desplazamiento de la Intervención Social del Estado hacia la pobreza y junto con esto la expansión de la asistencia. 2 - Al mismo tiempo y en relación a la creciente conflictividad y cuestionamiento social a las políticas de ajuste neoliberal y las consecuencias sociales generadas por estas, a las respuestas construidas por los gobiernos emergentes y organismos internacionales en este contexto de crisis procurando reconstruir la legitimidad.

En este proceso, se produce la institucionalización y consolidación de los PTRC como uno de los ejes centrales de los sistemas de protección social. Al mismo tiempo los propios organismos internacionales reconocen el agravamiento de la cuestión social, el aumento de los índices de la pobreza y confirman la necesidad estratégica de afrontar medidas que superen los acuerdos del consenso de Washington, habilitando un conjunto de recomendaciones teóricas prácticas y políticas que favorezcan la legitimidad del orden y la cohesión social. Las mismas apuntan a introducir reformas institucionales duraderas que atiendan en términos de alivio la extensa desprotección social desarrollada por la erosión estructural de las formas históricas de seguro y seguridad social de cada país. Junto con la erosión, retracción y debilitamiento del formato del seguro, se da la ampliación de formas de transferencia de renta de raíz no contributiva. Los PTRC se ubican en este movimiento general de recomposición de la protección social, sus dimensiones materiales y simbólicas, en un contexto de transformaciones radicales de la lógica de acumulación capitalista.

Se observa en la región un proceso de institucionalización de los PTRC, que implicó en cada país dinámicas particulares que parten del desarrollo de programas puntuales y de emergencia en contextos de crisis aguda, a programas que pretenden constituirse en permanentes vinculados a las estrategias globales de combate a la pobreza estructural más allá de situaciones de emergencia económica y social. Al mismo tiempo sus procesos de institucionalización implicaron la extensión y masificación de la asistencia, desbordando los estrechos límites de la hiper-focalización de la década del noventa. También este proceso de extensión de la asistencia a partir de los PTRC, implicó la ampliación de los programas sociales no contributivos, estableciéndose arreglos institucionales específicos en cada experiencia nacional en función de sus trayectorias históricas particulares $^{17}$

El análisis situado desarrollado nos permite comprender los fundamentos históricos- concretos de la emergencia de los PTRC y la centralidad que adquieren en los actuales formatos de protección social.

\section{CONCLUSIÓN}

Los sistemas de protección social constituyen una mediación institucional político- económica resultado al mismo tiempo de las contradicciones y reivindicaciones emanadas de las luchas de clases y de la lógica de acumulación capitalista. Es un proceso dinámico que se explica en el movimiento histórico de la sociedad. Esta perspectiva de totalidad nos permite comprender el sentido político y social de la política social, en relación a los procesos de acumulación, a los procesos de organización del trabajo y a la configuración de los sistemas de protección social. 
La protección social en América Latina, desde los años ochenta, viene evidenciando un desplazamiento hacia la focalización de sus acciones en la pobreza y extrema pobreza; desde acciones de emergencia hiper-focalizadas a programas institucionalizados de alcance masivo que penetran la arquitectura de protección social definida.

Pese a las mejoras registradas durante el siglo XXI, persisten grandes deudas, evidenciadas en la persistencia absoluta de la pobreza desde la década del ochenta y la permanencia de la estructura de desigualdad de la sociedad.

Si bien estas políticas se colocan en relación a la ampliación del consumo mínimo por parte de los sectores pobres de la población; ellas no afectan el origen de la pobreza y las desigualdades, porque la perspectiva de economía política en la cual se inscriben se basa en una concepción de desigualdad en relación a la noción de libertad para el acceso a los beneficios del mercado; al mismo tiempo la pobreza se explica en relación a la ausencia o insuficiencia de renta y no a las relaciones de desigualdad que la produce. Es decir prevalece el desafío de develar las raíces estructurales económicos y políticos de la pobreza, observando la producción de riqueza y las relaciones de poder en las cuales se asienta en la sociedad.

La creciente importancia asignada a la noción de capital humano, se centra en una concepción individualista de la sociedad. Imputan a los pobres un comportamiento que deben modificar para mitigar la pobreza, en tal sentido sostienen que la política social debe generar incentivos para que esos cambios se produzcan. Se exigen condicionalidades para la recepción de los bienes que implican la modelación de los comportamientos sociales. De esa forma los pobres son definidos por su situación de carencia económica y también por sus carencias actitudinales y formativas que los inhabilita para su desarrollo. En tal sentido la red de protección para pobres tendida, coloca esfuerzos en el desarrollo de habilidades o capacidades a través del entrenamiento educacional.

Si bien en la dimensión argumentativa de los PTRC, se incorporan nociones de derechos y el problema de la universalización; conviven en la práctica nociones minimalistas y procesos clasificatorios que reactualizan el tratamiento de la pobreza en términos de merecimineto/ no merecimiento, opacando la perspectiva de derecho social. Si bien la materialización de estos programas, contribuyen a volver menos dramáticas las condiciones de vida de este sector de la clase trabajadora que no puede reproducir sus condiciones materiales de existencia, y pretenden instalar una noción de derecho, de garantía y de universalización que es diferente al tratamiento dominante de la hiper-focalización de la década anterior y habilita potencialmente un camino de exigencia de cumplimiento de estas garantías.

El análisis de las políticas de transferencia particular en el marco de las formas históricas de protección social definida, nos permite comprender la existencia centralmente de un problema político: el proyecto de sociedad que se quiere construir. Se observa tanto las disputas en relación al reconocimiento de necesidades sociales, como las maneras de definir y organizar el trabajo. El resultado de estas disputas se materializa en las mediaciones político-económicas que condensan esas tensiones. Si aspiramos a una sociedad que se mueva en un sentido de la igualdad social, es necesaria una perspectiva amplia de la protección social, basada en un enfoque de derecho universal, que tienda a la superación de la segregación y fragmentación en las que históricamente han participado las políticas asistencialistas. El proceso en marcha muestra que si bien hay avances en la definición de un piso de protección, se está muy lejos de ese horizonte social.

\section{REFERENCIAS}

ANTUNES, R. El trabajo entre la perennidad y la superfluidad. Algunos equívocos sobre la deconstrucción del trabajo. In: SOTO, S. F.; TRIPIANA, J. (Comp.). Políticas sociales, trabajo y trabajadores en el capitalismo actual. Buenos Aires: Espacio, 2009.

CECCHINI, S. Aportes y limitaciones de los programas de transferencias condicionadas, Taller regional de capacitación: politicas orientadas a las familias para la reduccion de la pobreza y la conciliacion de la vida laboral y familiar en America Latina. Santiago de Chile, 2013.

COMISIÓN ECONÓMICA PARA AMÉRICA LATINA $Y$ EL CARIBE. Panorama Social de América Latina, 2011. Santiago de Chile, 2011.

Panorama Social de América Latina 2014. Santiago de Chile, 2014.

FILGUEIRA, F.. Tipos de welfare y reformas sociales en América Latina. Eficiencia, residualismo y ciudadanía estratificada. Social Science Research Council, Guadalajara, 1997. Versión revisada y modificada del documento presentado en el marco del proyecto Social Policy Citizenship in Central America.

Cohesión, riesgo y arquitectura de protección social en América. Santiago de Chile: CEPAL, 2007.

GAITÁN, F. Crecimiento, desigualdad y pobreza en el capitalismo periférico. Análisis de los países latinoamericanos. In: DI VIRGILIO, M; OTERO, M. P.; BONIOLO, P. (Coords.). Pobreza y desigualdad en américa latina y el caribe. Buenos Aires: 
CLACSO Libros, 2010. (Colección CLACSO-CROP). Disponible en:<www.clacso.org.ar $>$. Acceso en: 7 jun. 2015.

GOUGH, I. Economia política del Estado del bienestar. Traducción: Gerório Rodriguez Cabrero. Madrid: H.Blume Ediciones, 1978.

HARVEY, D. La condición postmoderna. 1. ed. Buenos Aires: Amorroutu, 2004.

El nuevo imperialismo. Madrid: Akal,

2007.

MANDEL, E. Marx y el porvenir del trabajo humano. Cuadernos de Mientras Tanto, Buenos Aires, n. 1, 1982.

MARX, K. El Capital. Buenos Aires: FCE, 1986. Tomo I.

MESA-LAGO, Carmelo. Social Security in Latin America. [S. I.: s. n.], 1991. Informe Report.

ORGANIZAÇÃO INTERNACIONALDO TRABALHO. Panorama Laboral 2011: América Latina y el Caribe. Lima, 2011. Disponible en:<www.oit.org.pe>. Acceso en: 7 jun. 2015.

REPETTO, F. Protección Social en América Latina: la búsqueda de una integralidad con enfoque de derechos. Revista del CLAD, [S. I.], n. 47, 2010. Disponible en:<www.clad.org>. Acceso en: 7 jun. 2015.

\section{NOTAS}

1 Esta ponencia, presentada en la VII Jornada Internacional de Políticas Públicas, São Luís, 25 a 28 de agosto de 2015, contiene resultados parciales de estudios desarrollados con apoyo de la Fundação Coordenação de Aperfeiçoamento de Pessoal de Nível Superior (CAPES) y del Conselho Nacional de Desenvolvimento Científico e Tecnológico (CNPq).

2 lan Gough (1978, p. 66) indica, el carácter contradictorio de la construcción de la intervención social del Estado, que atiende al mismo tiempo a los intereses de la acumulación y los procesos de protección social. "El Estado tiende a actuar con el fin de asegurar las condiciones que reproduzcan ese modelo y las relaciones de exploración dentro de él, que bajo el capitalismo significa asegurar la acumulación continua de capital [...] el Estado del Bienestar engloba una actitud racional que también se opone a la del mercado. En algún sentido actúa para 'satisfacer las necesidades' y extender los derechos y haciéndolo así, contradice los simples requerimientos directos del sistema de economía capitalista. [...] esta es, pues, la razón por la que nosotros caracterizamos el Estado del Bienestar como un fenómeno contradictorio."

3 Para una definición de las dimensiones a considerar en la diferenciación de los sistemas de protección social véase, Filgueira (1997).

4 Carmelo Mesa-Lago (1991), para realizar una diferenciación de las experiencias latinoamericanas, distingue los inicios históricos y grados de maduración de los sistemas de protección social, a partir de lo cual diferencia entre países pioneros, intermedios y tardíos. Esta clasificación destaca el momento en el cual cada país empezó a ocuparse de la protección social de sus habitantes por medio de sus políticas públicas. Observa sus procesos de maduración considerando las trayectorias históricas, la cobertura alcanzada, la relación con los modelos de desarrollo predominantes. Argentina, Uruguay y Brasil integran el grupo de países pioneros en la construcción de la protección social, dado que hacia los años '20 del siglo XX, los tres países registraban formas de desarrollo de medidas de protección social para su población.

5 Por ejemplo en los gobiernos de Perón en Argentina, Batlle en Uruguay y Vargas en Brasil.

6 De aquí surgen tres formatos de protección social: Universalismo estratificado. (Uruguay, Argentina, Chile), Regímenes duales (Brasil, México), y Regímenes excluyentes. (República Dominicana, Guatemala, Honduras, El Salvador, Nicaragua, Bolivia, Ecuador).

Sobre este proceso, ver Antunes (2009, p. 19) quien afirma: "En medio de tanta destrucción de fuerzas productivas, de la naturaleza y del medio ambiente, existe también una acción destructiva contra la fuerza humana de trabajo que tiene enormes contingentes precarizados 0 al margen del proceso productivo, elevando la intensidad de los niveles de desempleo estructural."

8 Los modelos que se desarrollan se basan en la perspectiva cepalina y los aportes de Raúl Prebish.

9 Una forma específica de política keynesiana configuró los modelos de desarrollo en América Latina entre 1930 y 1970-80, desarrollándose el modelo sustitutivo de importaciones, donde el estado asume un rol central en los procesos de planificación económico y social. El Estado financia el crecimiento de industrias orientadas a la producción para el consumo interno, a través de una política de subsidios y diversas medidas proteccionistas, sobre la base de la riqueza generada por productos primarios de exportación. Al mismo tiempo el estado incorpora mano de obra excedente, participando en la construcción de obras básicas de infraestructura económica y social.

10 Los cambios operados en las condiciones generales de producción se expresan en el incremento en volumen y cambios cualitativos en la composición de la masa trabajadora y explotada. Como tendencia general se hace observable la constitución de una masa de población sobrante para las necesidades de fuerza de trabajo del capital este movimiento tendencial de la sociedad capitalista es analizado en Marx (1986). Para un análisis contemporáneo donde se analiza la relación entre cambios tecnológicos, productividad del trabajo y tasas de ganancias, véase Mandel (1982).

11 La acumulación flexible "[...] apela a la flexibilidad con relación a los procesos laborales, los mercados de mano de obra, los productos y las pautas del consumo. Se define por la emergencia de sectores totalmente 
nuevos de producción, nuevas formas de producir servicios financieros, nuevos mercados y, sobre todo, niveles sumamente intensos de innovación comercial, tecnológica y organizativa." (HARVEY, 2004, p. 17071), implicando una reconfiguración espacio-temporal en el mundo capitalista.

12 Al mismo tiempo la pobreza afecta más a la niñez, las mujeres y las poblaciones indígenas en términos comparativos con otros sectores de la sociedad (REPETTO, 2010). Para el caso argentino véase: BERTRANOU, F. M.; BONARI, D. (Coords.). Protección social en Argentina. Santiago: Oficina Internacional del Trabajo, 2005

13 Las líneas de indigencia, que muestran el costo de adquirir una canasta básica de alimentos, se actualizan año a año según la variación del IPC de los alimentos, mientras que el componente no alimentario de la línea de pobreza se actualiza según la variación del IPC correspondiente.

14 Tomando la distribución del ingreso de manera desagregada, se observa que el ingreso del decil más pobre en los países de la región se ubica en torno al 1\%, en contraposición a los países europeos o asiáticos que es superior al $3 \%$. En el extremo opuesto, el decil más rico se apropia en América Latina de al menos el $40 \%$ de los ingresos (con casos extremos como Colombia o Brasil, cercanos al $50 \%$ ). Cifras que muestran la matriz profundamente desigual de distribución del ingreso en la región (GAITÁN, 2010).

15 Aun cuando los países latinoamericanos muestran grados distintos de concentración del ingreso, todos exhiben índices de Gini que superan al promedio de cada una de las regiones analizadas, exceptuando el África subsahariana (COMISIÓN ECONÓMICA PARA AMÉRICA LATINA Y EL CARIBE, 2011).

16 De acuerdo a las estimaciones más recientes del desempeño económico, el PIB regional crecerá en torno a $4.5 \%$ en 2011 respecto del año anterior. Encabezan esta expansión algunos países exportadores de materias primas de América del Sur más articulados con la demanda de las economías emergentes como Argentina, Chile, Paraguay, Perú y Uruguay, que crecerían alrededor de 6\% en 2011 (ORGANIZAÇÃO INTERNACIONAL DO TRABALHO, 2011).

17 Los PTC alcanzan para el 2013 a 30 millones de familias, equivalente a cerca de 127 millones de latinoamericanos y caribeños (CECCHINI, 2013). Los programas con mayor número absoluto de beneficiarios son: Bolsa Familia, en Brasil (13,8 millones de familias, 56,4 millones de personas), cobertura equivalente a $46 \%$ del total en la región; $y$, Oportunidades, de Mexico (6,5 millones de familias, 31,2 millones de personas), Familias en Accion, de Colombia (2,8 millones de familias, 11,7 millones de personas) y Bono de Desarrollo, en Ecuador (1,2 millones de familias, 6,4 millones de personas).

\section{Silvia Gabriela Fernández Soto}

Trabajadora Social

Doutora em Serviço Social pela Pontifícia Universidade Católica de São Paulo (PUC-SP)

Profesora Titular da Facultad de Ciencias Humanas (FCH) da Universidad Nacional del Centro de la Provincia de Buenos Aires (UNCPBA)

E-mail: silviafernandezsoto@gmail.com
Universidad Nacional del Centro de la Provincia de Buenos Aires - UNCPBA

Gral. Pinto 399, Tandil, Buenos Aires/Argentina 